\title{
Positive Pregnancy Test
}

National Cancer Institute

\section{Source}

National Cancer Institute. Positive Pregnancy Test. NCI Thesaurus. Code C92849.

A test result indicating the presence of hormones (HCG) in the blood or urine of a woman that confirm pregnancy. 